\title{
L'interprétariat en santé mentale : divisions sociale, morale et spatiale du travail dans les soins aux migrants
}

Interpretation in Mental Health Services: Social, Moral and Spatial Divisions of Work in Migrant Care

La interpretación en la salud mental: divisiones sociales, morales y espaciales en el trabajo de atención al migrante

\section{Anaïk Pian, Anne-Cécile Hoyez et Simona Tersigni}

\section{OpenEdition}

\section{Journals}

Édition électronique

URL : https://journals.openedition.org/remi/10768

DOI : $10.4000 /$ remi. 10768

ISSN : $1777-5418$

Traduction(s) :

Interpreting in mental health: Social, moral and spatial divisions of labor in health care for migrants URL : https://journals.openedition.org/remi/12095 [en]

Éditeur

Université de Poitiers

Édition imprimée

Date de publication : 1 octobre 2018

Pagination : $55-78$

ISBN : 979-10-90426-62-7

ISSN : 0765-0752

Référence électronique

Anaïk Pian, Anne-Cécile Hoyez et Simona Tersigni, « L'interprétariat en santé mentale : divisions sociale, morale et spatiale du travail dans les soins aux migrants », Revue européenne des migrations internationales [En ligne], vol. 34 - $n^{\circ} 2$ et 3 | 2018, mis en ligne le 01 janvier 2021, consulté le 14 avril 2022. URL : http://journals.openedition.org/remi/10768; DOI : https://doi.org/10.4000/remi.10768 


\title{
L'interprétariat en santé mentale : divisions sociale, morale et spatiale du travail dans les soins aux migrants.
}

\author{
Anaïk Pian ${ }^{1}$, Anne-Cécile Hoyez ${ }^{2}$ \\ et Simona Tersigni ${ }^{3}$
}

Au milieu des années 1980, des manuels français de médecine distinguaient trois types de pathologies singularisant les étrangers. On y parlait de pathologies dites d'importation, d'acquisition et d'adaptation à la société d'accueil, cette dernière catégorie faisant référence à des troubles psychiques (Fassin, 2000b). Dans ce modèle inculqué à des générations de médecins, le corps des étrangers, perçu comme "vecteur et récepteur passif de maladies" (ibid. : 5), était associé à un double risque : risque de contamination pour la société d'accueil et risque pour les migrants eux-mêmes, ces pathologies - et notamment celles psychiques - nuisant à leur "bonne intégration". Si les sciences sociales françaises ont tardé à aborder la question de la santé mentale en migration (Cognet et al., 2014), plusieurs études ont contribué à déconstruire les visions culturalistes qui, pendant longtemps, ont prévalu - et prévalent encore parfois - dans le milieu médical. À contre-pied des approches faisant de la culture et du "choc des cultures" le facteur explicatif des troubles psychiques, ces études ont montré comment ceux-ci s'imbriquent aux conditions de la migration (précarité, isolement, discriminations, etc.), tout en soulignant plus particulièrement les effets délétères de l'exil (Pestre, 2010; Veïsse et al., 2017) sur la santé mentale.

La prise en charge de cette population - notamment celle qualifiée d'allophone dans la terminologie institutionnelle actuelle ${ }^{4}$ - soulève aujourd'hui encore un enjeu fondamental : comment mettre en œuvre un suivi thérapeutique en l'absence d'une langue commune entre soignants et patients? L'oralité et la mise en parole sont en effet au cœur du dispositif d'écoute, qu'il s'agisse

\footnotetext{
1 Sociologue, Maitre de conférences, DynamE, Université de Strasbourg-CNRS, Bât. 5, Le Patio, 22 avenue René Descartes, 67000 Strasbourg ; pian@unistra.fr

2 Géographe, Chargée de recherche CNRS, ESO, Université de Rennes-CNRS, Maison de la Recherche en Sciences Sociales, Place du Recteur Le Moal, 35043 Rennes Cedex; anne-cecile.hoyez@univ-rennes2.fr
}

3 Sociologue, Maitre de conférences, SOPHIAPOL, Université Paris Ouest Nanterre, 200 avenue de la République, 92001 Nanterre ; stersigni@parisnanterre.fr

4 Cette catégorie est notamment mobilisée de manière très explicite dans l'éducation nationale. Voir Armagnague-Roucher et al. (2018), ainsi que Armagnague-Roucher et Tersigni (2019). 
de détecter des symptômes, d'établir un diagnostic ou d'effectuer les soins impartis. Dès lors que l'on s'intéresse, en contexte migratoire, aux prises en charge en santé mentale, la question de l'interprétariat s'avère ainsi constituer une dimension centrale.

Le défaut d'interprétariat participe des inégalités sociales de santé et d'accès aux soins : plusieurs travaux ont souligné combien la barrière de la langue constitue un obstacle majeur aux soins (Kotobi et al., 2013), qu'ils soient somatiques ou psychiques. Pendant longtemps en France, l'interprétariat professionnel a eu du mal à s'imposer dans le monde médico-social (Tabouri, 2009; Pian, 2014), en dépit de recommandations émanant de cadres réglementaires nationaux et européens. Cette difficile institutionnalisation est à resituer dans un contexte national républicain ${ }^{5}$ qui, ne reconnaissant pas les minorités en tant que telles, est réticent à mettre en place des dispositifs spécifiques à leur égard. Cependant, l'interprétariat professionnel connaît aujourd'hui un moment charnière dans le domaine médico-social. La nouvelle loi "santé " de janvier $2016^{6}$, dont le décret d'application ${ }^{7}$ a été publié en mai 2017, a inscrit dans les pratiques des établissements publics des mesures promouvant la médiation sanitaire et l'interprétariat linguistique, en particulier pour les populations éloignées du système de santé. Bien que ces mesures ne soient ni contraignantes ni accompagnées de moyens financiers, elles renforcent les démarches d'associations cherchant à faire reconnaître ce métier au cœur du système de santé. Elles appuient également les recommandations du Plan psychiatrie et Santé mentale (2011-2015) quant au recours à l'interprétariat professionnel dans les suivis thérapeutiques des patients ne parlant pas ou peu français.

Or, dans le même temps, l'interprétariat interpelle l'étiologie (Rhenter et Michel, 2010; Coldefy, 2016) des prises en charge. La présence d'un interprète remet en cause le colloque singulier soignant/soigné auquel certains courants psychiatriques sont très attachés. En revanche, d'autres courants, comme l'ethnopsychiatrie, qui ne fait pourtant pas I'unanimité en termes d'affranchissement de la pensée coloniale (Fassin, 2000a), conçoit la présence de l'interprète comme co-actrice du soin. En France, dès le début des années 1960, l'interprétariat a été au centre des interrogations de la pratique thérapeutique dans le centre

5 De nombreux travaux en sciences sociales ont permis d'interroger les effets d'une "tradition" nationale républicaine se prétendant universaliste et produisant pourtant, en son sein, des catégorisations ethnicisantes et racisantes, tant sur le plan institutionnel que dans le sens commun (De Rudder et al., 2000; Simon, 2006; Fassin, 2012; Crenn et Kotobi, 2012).

6 Dont l'article 90 souligne la nécessité d' «améliorer l'accès aux droits, à la prévention et aux soins des personnes éloignées des systèmes de prévention et de soins, en prenant en compte leurs spécificités ".

7 Selon l'art. D. 1110-6 de ce décret d'application, "L'interprétariat linguistique dans le domaine de la santé désigne la fonction d'interface, reposant sur des techniques de traduction orale, assurée entre les personnes qui ne maîtrisent pas ou imparfaitement la langue française et les professionnels intervenant dans leur parcours de santé, en vue de garantir à ces personnes les moyens de communication leur permettant d'accéder de manière autonome aux droits prévus au présent titre, à la prévention et aux soins. L'interprétariat linguistique dans le domaine de la santé garantit aux professionnels de santé les moyens d'assurer la prise en charge des personnes qui ne maîtrisent pas ou imparfaitement la langue française dans le respect de leurs droits prévus au présent titre, notamment du droit à l'information, du droit au consentement libre et éclairé, du droit au respect de leur vie privée et au secret des informations les concernant». 
Françoise Minkowska (Bourdin et Larchanché, 2015). Plus tard, des praticiens se sont interrogés sur la manière de retrouver les catégories du repérage sémiologique et de la thérapeutique psychiatrique dans les séances thérapeutiques avec des réfugiés originaires d'Asie du Sud-Est qui, à ce moment-là, sont définis en tant que "non francophones" par l'institution psychiatrique (Rechtman, 1992 : 347). Derrière les débats sur les configurations du soin en situation d'interprétariat, c'est aussi la conception de l'Autre comme patient étranger qui fait débat, renvoyant à des représentations tenant à I'histoire même des professions et des formations médicales ${ }^{8}$.

Depuis les années 2000, en outre, la situation de précarité économique et surtout administrative d'une partie du "public migrant " ${ }^{9}$ (Hachimi et Nacu, 2010; Le Défenseur des droits, 2016) interpelle de plus en plus la psychiatrie (Chambon et Le Goff, 2016). Ces dernières années, des publications spécialisées (Cahiers du Rhizome, 2015; L'information psychiatrique, 2015a, 2015b, 2015c; Psychologie clinique, 2017) questionnent la nécessité d'outiller la "clinique de l'exil » dans un contexte où le "politique travaille et met à mal l'intime" au point de nécessiter "la reconstruction d'un espace d'interlocution psychique, entre la personne et ses thérapeutes" (Piret et Douville, $2017: 6$ ).

Dans ce contexte, l'article interroge, à travers le prisme de l'interprétariat, la division sociale, morale et spatiale du travail dans la prise en charge des migrants ${ }^{10}$ en santé mentale. La notion de "division sociale et morale du travail ", empruntée à Hughes (1958) et reprise par Payet (1997), rend compte des écarts, répartitions et tensions traversant les rapports entre des professionnels amenés à travailler ensemble au regard d'une part, des tâches imparties et celles effectivement réalisées, et d'autre part, au regard de leur prestige et hiérarchisation. Alors que les membres d'une profession ont intérêt à faire croire en la noblesse de leur activité, Hughes (1958) souligne qu'ils doivent s'arranger avec des tâches nécessaires et moins prestigieuses, qui plus est se déroulant souvent sous le regard d'autrui. Ce qui est spécifique à l'interprétariat en santé mentale, c'est que la relation de soin doit composer avec un interprète, au service du praticien, mais aussi du patient, par lequel sont transportées les mises en mots.

L'orientation pluridisciplinaire adoptée, combinant regard sociologique et géographique, invite cependant à élargir la notion de division sociale et morale

8 Cette histoire est notamment marquée par des discours et pratiques rejetant du système médical les médecins étrangers ou formés à l'étranger (Hassenteufel, 2008). Par ailleurs, depuis plusieurs décennies, la formation à I'anthropologie ou à I'ethnologie voire à la "connaissance des cultures" ou aux "soins interculturels" s'inscrit dans un mouvement promouvant l'humanisation des soins, et devant conjointement permettre au patient de devenir un acteur "autonome" et "responsable" (Kessar, 2000). Toutefois, l'auteure montre comment les difficultés rencontrées dans les prises en charge ne relèvent pas tant d'un manque de connaissances sur la culture d'autrui à l'hôpital, mais plus largement de la configuration des prises en charge, marquée notamment par un "manque de communication sur le patient entre professionnels" (ibid.).

9 Catégorisation englobante, notamment utilisée par les professionnels du secteur médico-social, et qui renvoie à une pluralité de statuts (demandeurs d'asile; irréguliers; réfugiés, etc.). Les patients ainsi désignés ont pour point commun d'être étiquetés comme vulnérables et de vivre en situation de précarité.

10 Nous avons fait le choix de ne pas indiquer le féminin au pluriel des mots, migrant·e·s et professionnel-le.s. Toutefois, la dynamique des rapports sociaux de sexe est présente en consultation, et ce, autant entre patients et soignants, qu'avec l'interprète. 
du travail en y adjoignant la dimension spatiale. En effet, les pratiques des professionnels sont traversées par des enjeux relatifs aux territoires d'exercice et de vie des patients. En prenant en compte ces variations, il s'agira de resituer, au sein des deux villes de l'enquête, Rennes et Strasbourg, la répartition des prises en charge des migrants dans différents lieux. Cette approche, qui invite à "prendre en considération la contingence des configurations socio-spatiales" (Bonny et al., 2017), permet de penser les faits sociaux et politiques à travers l'émergence de dynamiques localisées.

L'article est organisé en trois parties. La première présente les enjeux liés à la professionnalisation de l'interprétariat médico-social, ce qui est nécessaire pour recontextualiser les tensions repérées dans la division sociale, morale et spatiale des prises en charge. La seconde partie met en lumière la manière dont les besoins, le recours (ou l'absence de recours) aux ressources locales en interprétariat par les structures ainsi que par les professionnels de la santé mentale marquent le paysage sanitaire. Centrée sur la dynamique des consultations ${ }^{11}$, une troisième partie confronte le point de vue des interprètes sur leurs pratiques à celui des psychiatres et psychologues recourant à leurs services. En articulant ces différentes dimensions, ce texte éclaire la manière dont les interprètes en tant que "nouveaux acteurs du soin" viennent (re) composer la configuration de prise en charge des migrants.

\title{
Encadré 1 : Méthodologie
}

\begin{abstract}
L'article s'appuie sur un terrain mené dans deux capitales régionales ${ }^{12}$. En Alsace, il a été réalisé à Strasbourg; en Bretagne, il a été effectué à Rennes et dans sa première couronne. Outre le décentrement du regard sur des terrains encore peu investis, ce choix conjugue plusieurs intérêts. En effet, Strasbourg et Rennes sont des villes phares dans l'impulsion d'initiatives locales promouvant le développement de l'interprétariat professionnel. Ces initiatives sont notamment portées par les actions de Migrations santé Alsace (MSA) à Strasbourg et du Réseau Louis Guilloux (RLG) à Rennes. Ces deux associations sont engagées dans des négociations et des partenariats avec les collectivités locales et les instances de santé (notamment les Agences régionales de santé). Enfin, bien que Rennes et Strasbourg aient une histoire migratoire distincte (ancienne pour l'Alsace, plus récente pour la Bretagne), elles sont toutes les deux marquées depuis les années 2000 par une évolution des flux migratoires. Elles connaissent en particulier une présence de plus en plus importante de demandeurs d'asile (et de déboutés) en provenance des pays de l'ex-bloc soviétique et d'Afrique. Entre 2014 et 2015 - d'après les sources de l'Office français des réfugiés et des apatrides et du ministère de l'Intérieur, employées dans la synthèse statistique produite par l'Observatoire régional de l'intégration et de la ville en 2017 pour la région Grand Est, les titres pour raisons humanitaires (réfugiés, bénéficiaires de la protection subsidiaire notamment) ont connu l'accroissement le plus important, en comparaison avec les titres de séjour délivrés pour raisons familiales $(37,6 \%)$ ou études
\end{abstract}

11 L'étude ne traite pas des consultations destinées aux enfants, bien que ce terrain mériterait d'être creusé.

12 Le terrain est réalisé dans le cadre de deux projets de recherche : le projet PEPS du réseau national des MSH - IMSODA (2016-2017) et I'ANR MIGSAN (2016-2019). 
(34,2 \%). En 2016, le guichet unique du Bas-Rhin a enregistré 1634 premières demandes d'asile, et celui d'Ille-et-Vilaine 1724 (OFII, 2017). Ce dernier chiffre marque également la hausse de la demande d'asile en Bretagne puisqu'entre 2012 et 2014, une moyenne annuelle de 1200 nouvelles demandes a été déposée dans la région (Plateforme d'accueil des demandeurs d'asile, 2016). À Rennes, par ailleurs, en 2015, 342 demandeurs d'asile ont consulté dans le centre médical du RLG, dont plus de la moitié étaient originaire d'Afrique subsaharienne (République démocratique du Congo, Soudan, Somalie). L'étude repose sur une démarche qualitative. Elle mobilise des entretiens (cinquante-six au total) auprès d'interprètes professionnels salariés de MSA et du RLG (trente-neuf), de responsables de ces associations engagés dans la professionnalisation de l'interprétariat (quatre), et de professionnels de la santé mentale (treize). Les interprètes professionnels interrogés officient en arabe, berbère, russe, géorgien, arménien, italien, serbo-croate bosniaque, albanais, turc, kurde, anglais. Au moment des entretiens, leur expérience dans le métier pouvait être très récente (quelques mois) ou beaucoup plus longue, de quelques années à plus d'une quinzaine d'années. Les professionnels de la santé mentale interrogés exercent lou ont exercé) en tant que psychologues et psychiatres (médecins et infirmiers) en centre hospitalier, CMP (centre médico psychologique), EMPP (équipe mobile psychiatrie précarité), mais aussi dans des dispositifs de prise en charge intersectoriels associatifs. Ceux ayant accepté de nous rencontrer ont une expérience de travail plus ou moins ancienne avec les interprètes et tendent à défendre l'importance de l'interprétariat pour le suivi thérapeutique. Nous n'avons pu, cependant, mener des observations directes lors de consultations médicales, nos demandes auprès des professionnels de santé mentale s'étant heurtées à l'argument du secret médical. À défaut, des observations ont été effectuées lors de formations à destination d'interprètes et de soignants, que nous avons dispensées à la demande des associations concernées.

\section{L'interprétariat médico-social face à des enjeux de professionnalisation}

Rendre compte des enjeux soulevés par la division sociale, morale et spatiale du travail introduite ou accentuée par l'interprétariat dans la prise en charge des migrants nécessite de présenter rapidement les deux associations dans lesquelles les interprètes rencontrés sont salariés. II s'agira également d'éclairer leur profil sociologique, afin de mieux saisir les intentions de professionnalisation liées à la pratique.

Basé à Rennes, le Réseau Louis Guilloux (RLG) est une structure associative principalement financée par l'Agence régionale de santé de Bretagne. II comporte un pôle "migrant" (comprenant un centre de santé : le Centre Médical Louis Guilloux), un pôle interprétariat, et porte le projet DATASAM (Dispositif d'appui technique pour l'accès aux soins des migrants - un programme de régionalisation de l'accès aux soins du "public migrant précaire»). Le pôle interprétariat a commencé à être mis en place au milieu des années 2000 en même 
temps que l'équipe mobile psychiatrie précarité (EMPP) ${ }^{13}$. II s'est consolidé à partir de 2010, notamment autour des partenariats formés à l'échelle nationale avec les associations proactives pour la reconnaissance de la profession d'interprète médico-social. II met à disposition des structures demandeuses, sur le territoire rennais principalement et breton parfois, un service d'interprétariat "physique" (déplacement de l'interprète sur le lieu du rendez-vous) et éventuellement téléphonique. En 2015, le pôle interprétariat salarie dix-neuf interprètes couvrant vingt-deux langues, pour un volume horaire total de 9436 par an (Rapport d'activités RLG, $2016: 27$ ).

Créée en 1975 sous le nom de Groupe Médico-social d'Aide aux Migrants ${ }^{14}$, MSA (Migrations Santé Alsace) est une association engagée dans la promotion de l'accès aux droits et aux soins des populations migrantes. Elle dispose d'un important pôle interprétariat, qui constitue sa principale activité : en 2016, elle compte quatre-vingt-cinq interprètes actifs dans trente-huit langues, pour un total de 26023 heures d'interprétariat réalisées sur l'année dans le Bas-Rhin et le Haut-Rhin ${ }^{15}$ (Rapport d'activités MSA, 2016 : 18).

Les prestations en interprétariat dans le secteur de la santé mentale représentent une part importante de l'activité menée par MSA et par le RLG. Au sein des interventions réalisées par les interprètes de MSA en milieu hospitalier, la psychiatrie est de loin le secteur le plus consommateur en interprétariat (pour I'année 2016, 6372,5 heures d'interventions ont été réalisées). Les interventions au sein de I'EPSAN (Établissement public de santé Alsace Nord, structure clé de la santé mentale) représentent, quant à elles, $24 \%$ des prestations en interprétariat en milieu hospitalier (Rapport d'activités MSA, 2016 : 21-22). Pour le RLG, la psychiatrie représente le troisième poste de consommation (après le domaine médical - activité du centre médical Louis Guilloux - et le domaine social activité auprès des centres d'accueil pour demandeurs d'asile). Les interventions auprès du CHGR (centre hospitalier spécialisé-psychiatrie) sont réparties entre I'activité hospitalière, les CMP et I'EMPP.

\section{Un métier très ethnicisé et féminisé}

Le profil des interprètes recrutés à MSA et au RLG présente de grandes similitudes. Tout d'abord, I'interprétariat médico-social fait partie de ces métiers très féminisés, comme tend à l'être plus généralement le secteur du social (Bessin, 2005) : en 2015 à MSA, soixante-cinq femmes travaillent en tant qu'interprètes salariées contre seize hommes. Au RLG, quatorze interprètes sur dix-neuf sont des femmes. Par ailleurs, dans les deux associations concernées, la grande majorité des interprètes n'est pas née française et a connu une expérience migratoire (pour études ou raisons familiales) ou d'asile, certains ayant obtenu le statut de réfugié, d'autres ayant été déboutés puis régularisés. Plusieurs d'entre eux connaissent ou ont connu une situation précaire sur le plan du séjour; ils partagent en ce sens une certaine proximité sociale avec les usagers allophones qui, eux-mêmes, sont souvent confrontés à une précarité administrative.

13 Celle-ci est rattachée au centre hospitalier Guillaume Régnier, établissement public de santé mentale.

14 C'est en 1988 que l'association change de nom pour devenir Migrations Santé Alsace. 15 Notons que MSA dispose également d'une antenne à Mulhouse. 
L'entrée dans le métier d'interprète, qui se fait très souvent par les réseaux du bouche-à-oreille, constitue pour certains le premier emploi occupé, notamment durant ou à l'issue des études. D'autres ont connu des expériences professionnelles antérieures, dans des domaines tels que l'enseignement, les langues, le commerce, le sanitaire ou le social. Les emplois occupés auparavant en France ont souvent été vécus comme un déclassement par rapport au niveau d'étude effectué ou aux expériences professionnelles exercées au pays d'origine : "Je cumulais tous les handicaps, l'âge, l'origine. Personne ne voulait de moi. Ici l'origine est un avantage. L'interprétariat, pour dire vrai, au départ, ce n'est pas un choix", souligne ainsi une interprète $(\mathrm{Bac}+5)$, ayant commencé à exercer vers la cinquantaine. Si le métier d'interprète demeure un travail précaire ${ }^{16}$, I'origine (réelle ou supposée) y est souvent autoperçue comme une ressource potentielle, et moins comme un facteur de discrimination.

Or en l'absence, dans la très grande majorité des cas, de diplômes dans le domaine de l'interprétariat et/ou la traduction ${ }^{17}$, les savoir-faire des interprètes relèvent d'abord de savoir-faire biographiques. Non seulement ils officient le plus souvent dans leur langue maternelle, mais de plus, nombre d'entre eux ont été amenés, y compris parfois depuis leur plus jeune âge, à accompagner des membres de leur entourage dans des démarches administratives et de soins en leur servant d'interprète. D'autres ou parfois les mêmes ont une expérience d'interprète bénévole dans des associations d'aide aux étrangers. Dans ce contexte, les associations recrutant et salariant les interprètes ont progressivement engagé un travail de professionnalisation de la fonction.

Par professionnalisation, il est fait référence aux processus «de reconnaissance et d'autonomie de catégories de travailleurs et de leur stabilisation et légitimation comme groupes professionnels" (Demazière, 2009 : 85). Si les conditions d'apparition et d'utilisation du mot professionnalisation structurent des enjeux qui, en fonction des métiers, ont pu susciter des débats sociaux considérables (Wittorski, $2008: 9$ ), dans le cas des interprètes, I'on retrouve des enjeux communs à d'autres activités. II s'agit notamment de la volonté d'un groupe d'individus partageant la même activité de s'organiser sur le marché, mais aussi de l'intention des institutions, des organisations et des associations d'accompagner la flexibilité du travail. Ces processus - souvent conflictuels ou du moins concurrentiels - impliquent en outre une définition normative et pratique des frontières du métier. Cette dynamique a impulsé, à différents niveaux, un travail de codification de ce que doit être le métier d'interprète et comment il doit être exercé. Ces initiatives peuvent être vues comme relevant d'un processus visant à revendiquer un "mandat/mandate» au sens d'une mission reconnue (Hughes,

16 De nombreux interprètes sont en contrat CDII (contrat à durée indéterminée intermittente) avec un volume d'heures modulables et annualisées. Beaucoup exercent en parallèle une autre activité salariée (vacations diverses, emploi dans la restauration, l'aide à domicile, etc.). Malgré la précarité salariale, travailler à temps partiel, tout en pouvant indiquer à l'association ses plages d'indisponibilité, est parfois perçu comme un avantage permettant de concilier I'exercice du métier avec une vie familiale, des études en cours, etc. Plusieurs interprètes salariés exercent par ailleurs de manière concomitante comme interprètes/traducteurs assermentés auprès des tribunaux ou des commissariats.

17 À MSA et au RLG, le niveau d'études des interprètes est majoritairement situé entre bac +3 et bac +4 . Le niveau d'études exigé est moins important lorsqu'il est difficile de trouver un interprète dans une langue donnée. 
1958) qui, tout en valorisant l'activité menée, la distingue de potentiels concurrents (interprètes bénévoles, membres familiaux, auto-entrepreneurs).

À l'échelle nationale, la constitution d'un groupe de travail regroupant huit associations (dont MSA et le RLG) a donné lieu, en 2012 à Strasbourg, à I'adoption de la Charte de l'interprétariat médico-social professionnel en France ${ }^{18}$. Cette charte, novatrice, pose des jalons concernant la déontologie, les responsabilités des interprètes et les délimitations de leur pratique. En 2016, suite à la nouvelle loi santé promouvant l'interprétariat linguistique, ces associations ont été sollicitées par la Haute Autorité de Santé pour participer à la construction de référentiels et des règles de bonnes pratiques en matière d'interprétariat. La démarche de plaidoyer qu'elles ont initié collectivement dès le milieu des années 2000, a abouti sur une reconnaissance par les instances nationales de leur légitimité à être force de proposition dans ce domaine. Dans une démarche qui se révèle in fine être "bottom up", ce sont elles qui ont tout écrit du référentiel-métier, susceptible d'être entériné par le ministère (Arend et al., 2017).

Or, une fois actée la reconnaissance de l'interprétariat au niveau juridique, se poursuit une discussion sur la délimitation des compétences des interprètes dans leur collaboration avec les professionnels du soin. En interne, les associations promouvant l'interprétariat professionnel continuent d'engager un travail de codification du métier, d'autant plus que les interprètes sont amenés à exercer dans une pluralité de structures (centres d'accueil pour demandeurs d'asile, hôpitaux, CMP, etc.), avec des interlocuteurs divers et des attentes parfois différentes. La mise en place de groupes d'analyse de pratiques, de dispositifs de tutorat ou encore le renforcement de la formation continue marquent la volonté des associations d'ordonner la division sociale et morale du travail entre les différents acteurs. II n'en demeure pas moins que ces ressources en interprétariat construites et mobilisées grâce à des partenariats à l'échelle nationale sont, dans leur mise en œuvre locale, activées de façons différentes.

\section{Territorialisation des prises en charge : une organisation différenciée entre Rennes et Strasbourg}

Les structures et les professionnels en santé mentale n'ont pas tous les mêmes modes d'opérationnalisation des supports en interprétariat sur leur territoire. Ils composent avec les forces vives en présence (souvent en lien avec le secteur médical, médico-social et social) et avancent en fonction des débats et négociations entre les différents acteurs de la scène locale. De plus, ils se retrouvent à mettre en œuvre de nouvelles pratiques autour de l'interprétariat dans un domaine de la santé publique marqué par une territorialisation en secteurs psychiatriques, indépendants des autres découpages territoriaux.

18 Les autres associations sont I'ADATE (Grenoble), APTIRA (Angers), ASAMLA (Nantes), COFRIMI (Toulouse), ISM (antennes de Lyon et Paris), MANA (Bordeaux). 


\section{L'impact de la sectorisation psychiatrique sur l'interprétariat}

En France, la psychiatrie et la santé mentale répondent à une organisation calée sur un processus de territorialisation ${ }^{19}$ à un double niveau. Tout d'abord, le domaine répond à la sectorisation psychiatrique (héritée des réformes de 1960, puis transformée en 2009) qui définit ses missions et son organisation ${ }^{20}$. Ensuite, il s'inscrit dans les programmes d'aménagement du territoire appliqués par les Agences régionales de santé. II résulte de ce double périmétrage territorial et institutionnel une grande complexité dans la coordination des parcours de soins des patients. Le secteur de la psychiatrie répond à une territorialisation spécifique (héritée de son fonctionnement "exclusif») qui s'efface progressivement pour venir croiser les territoires des politiques de santé et du social. D'une position "de niche" (territoriale et professionnelle), les professionnels de la santé mentale transitent alors vers une mise en conformité avec les orientations des Agences régionales de santé, en renforçant le travail partenarial avec les sphères médicales, médico-sociales, sociales (Rhenter et Rhenter, 2012). Dès lors, la "philosophie de la sectorisation" (ibid.) leur impose de composer avec ces autres éthiques professionnelles dans une régulation multi-niveaux ${ }^{21}$.

Par ailleurs, s'il est un échelon où le secteur de la santé mentale évolue le plus en France, c'est au niveau local. La territorialisation de l'action publique en santé et en santé mentale s'effectue de plus en plus à micro-échelle : les notions de "proximité", "concertation" ou "partenariat" orientent de plus en plus les psychologues et psychiatres vers des formes d'organisation en "comités", en "équipes" ou en "réseaux" qui conduisent à mettre en place des dispositifs spécifiques (équipes mobiles psychiatrie précarité [EMPP], conseils locaux en santé mentale [CLSM], groupes d'entraide mutuelle [GEM]) branchés sur les dynamiques locales). Ces dynamiques, dépendantes de logiques propres à chaque site, sont hétérogènes dans le territoire national et les territoires régionaux : comme le soulignent Coldefy et Le Neindre (2014: 51), elles sont "à mettre en relation, d'une part, avec les traditions d'accueil [...] et, d'autre part, avec les politiques à l'œuvre et les initiatives des acteurs locaux ". Nous en avons fait le constat sur nos terrains où les EMPP et les CMP sont en première ligne dans la prise en charge des soins en santé mentale pour les migrants, avec en appui deux structures d'interprétariat professionnel en santé.

19 À travers la notion de territorialisation, nous entendons prendre en compte à la fois I'action publique et les processus de gouvernance et de gouvernement qui recomposent les systèmes d'action à différentes échelles territoriales (Eliot et al., 2017).

$20 \mathrm{La}$ notion de "secteur" en psychiatrie est intervenue en 1960 dans le mouvement de désinstitutionnalisation des soins psychiatriques. Le "secteur" répond à un découpage territorial indépendant des autres maillages administratifs dans lequel les professionnels du secteur public et privé s'engagent à mettre en place une coordination du parcours de soins dans sa globalité (prévention, soins, réinsertion sociale). Cela implique un travail pluridisciplinaire destiné à prendre en charge les patients "au plus près de leur espace de vie" (Coldefy, 2016).

21 La loi de janvier 2016 confirme l'existence de la psychiatrie de secteur, mais elle positionne aussi la psychiatrie dans la politique globale de santé mentale, qui ne contient pas exclusivement des soins psychiatriques (Coldefy, 2016). 


\section{L'interprétariat au prisme de la répartition des soins en santé mentale à Rennes}

À Rennes, l'EMPP est créée en 2002 à la suite du repérage sur le terrain de "nouveaux enjeux de santé mentale liés à la souffrance et la précarité" comme nous le disent le psychiatre et les psychologues lors d'un échange collectif fin 2016. L'EMPP était alors conçue comme un "nouvel outil» permettant d' " aller vers" les personnes en condition de précarité (Hoyez, 2015), puis les intégrer au droit commun. L'équipe rennaise a très vite été confrontée au phénomène migratoire et, de fait, à une clinique singulière sous certains aspects : selon celle-ci, quand, pour les autochtones, la précarité démarre dès l'enfance, elle devient surtout, pour les migrants en situation administrative précaire, un problème de statut administratif, de précarité résidentielle et de difficulté globale à aller vers le droit commun.

À Rennes, I'historique local de la territorialisation des secteurs de la psychiatrie se révèle très spécifique. En effet, le territoire d'action de l'EMPP couvre quarante-sept communes (soit environ 500000 habitants). Lorsque l'EMPP reçoit un patient, hormis le travail de prise en charge de première ligne, elle doit faire en sorte de I'aiguiller vers un CMP situé sur l'un des douze secteurs de leur territoire d'action couvrant Rennes, Fougères, Vitré et Redon. Comme partout en France, les personnes ayant un domicile établi sont suivies dans le CMP de leur secteur de résidence. En revanche, l'orientation des personnes non domiciliées (ou dites SDF) est plus singulière dans la mesure où elle est effectuée suivant leur mois de naissance. Ainsi, les personnes nées en janvier (01) seront suivies dans le secteur G1; les personnes nées en mars (03), dans le secteur G3; les personnes nées en mai (05) sur le secteur G5 et ainsi de suite. Cette répartition est héritée des débats qui ont suivi les réformes territoriales ayant touché I'Illeet-Vilaine, et que le psychiatre de l'EMPP résume ainsi :

"Tout a été redécoupé il y a une dizaine d'années à peu près avec simplement trois territoires rennais et des territoires ruraux. Sauf que l'affaire des dates de naissance n'a pas été changée, ce qui fait que ça bloque totalement par rapport à la précarité. Et puis personne ne veut changer le système parce que ça veut dire que les CMP de Rennes accepteraient de voir tous les SDF de Rennes à condition de récupérer les moyens des autres... et les autres ne veulent pas lâcher leurs moyens. Donc c'est bloqué. Et puis personne ne court après ces patients de toute façon." (Entretien, 2016)

Cette répartition - qui pose à la fois la question des moyens alloués et du type de public pris en charge - est considérée comme imparfaite par une grande partie des soignants rencontrés. Elle donnerait lieu à des incongruités totales, comme le souligne à nouveau le même psychiatre :

"Un migrant qui doit être suivi pour un syndrome post-traumatique, qui serait ici à Rennes, s'il est né au mois de mars, il doit partir, théoriquement, à Fougères. Alors en pratique, il y a possibilité d'avoir des suivis au bloc, en hôpital psychiatrique, ici, au bureau du psychiatre. Mais ce n'est pas une prise en charge multidisciplinaire d'un CMP. C'est juste un psychiatre qui fait les consultations. II n'y a pas de psychologue. Pas de suivi infirmier. En gros, c'est de la prescription d'antidépresseurs tous les deux mois, renouvellement de psychotropes." (Entretien, 2016). 
Du fait de cette répartition, I'EMPP rencontre aussi des difficultés à orienter ses patients vers les CMP hors de Rennes et se retrouve à faire quatre fois plus d'actes que les CMP (tous CMP confondus). De même, les CMP situés sur Rennes font plus d'actes que les autres CMP du territoire, en raison de leur proximité géographique avec le public précaire non domicilié, parmi lesquels se trouvent les migrants. Par ailleurs, dans la mesure où les migrants constituent la moitié de l'activité de I'EMPP, I'équipe a été saisie très tôt après sa création par le Réseau Louis Guilloux. Pour ces deux structures animées par des intérêts convergents, c'est une coordination de long terme qui se met en place depuis 2006, notamment autour de l'interprétariat professionnel.

\section{L'interprétariat au prisme de l'organisation des soins psychiatriques à Strasbourg}

À Strasbourg, à la différence de Rennes, existe une PASS (permanence d'accès aux soins de santé) psychiatrique, située en centre-ville, dans les mêmes locaux que le CMP (centre médico-psychologique) Berne et I'EMPP (équipe mobile psychiatrique précarité, relevant d'un dispositif national), toutes deux dépendantes de I'EPSAN (établissement public de santé Alsace Nord).

La PASS et l'EMPP prennent en charge des patients en situation de précarité. La PASS accueille les patients sans couverture médicale, qu'ils soient sansabris, primo-arrivants, déboutés du droit d'asile ou plus largement en situation irrégulière. Les soignants tendent à distinguer les patients non étrangers avec des troubles psychiatriques (psychoses, psychosomatismes, addictions), des étrangers présentant des troubles liés aux conditions de la migration et de l'exil, avec ou sans troubles associés. Selon les médecins coordinateurs qui se sont succédé à la PASS psychiatrique depuis son ouverture au milieu des années 2000, une sensibilité plus ou moins importante a été accordée au "public migrant». Lorsque les patients bénéficient, avec l'aide du service social de la PASS, d'une ouverture de droits, ils continuent à être suivis par la même équipe de l'EMPP, dès lors qu'ils sont sans-abris ou déclarent l'être. En effet, par crainte des autorités publiques, certains migrants sans titre de séjour n'osent communiquer leur adresse (même s'ils en ont une) aux personnels soignants. En revanche, ceux qui peuvent faire valoir un logement sont susceptibles d'être orientés vers le CMP de leur secteur. Celui-ci est défini en fonction de leur adresse de domiciliation. Par ailleurs, il est fréquent qu'à l'ouverture des droits, des migrants disposent d'une domiciliation, mais considérée comme non "stable" par les équipes : c'est notamment le cas lorsqu'ils sont hébergés dans des structures d'urgence sociale ou qu'ils sont domiciliés dans des centres d'accueil pour demandeurs d'asile ou par des associations de soutien aux étrangers. S'ils ont des enfants scolarisés en maternelle ou en élémentaire, la réorientation vers le CMP de secteur est alors effectuée sur la base de l'adresse de l'école, retenue comme critère de sectorisation. En l'absence d'enfants scolarisés en primaire, les patients continuent d'être suivis au CMP Berne. Cependant, les réorientations peuvent donner lieu à des arrangements internes, afin de garantir une répartition de la charge de travail sur le territoire de l'Eurométropole et d'éviter un engorgement des listes d'attente. Les choix ainsi opérés dépendent aussi de l'urgence de la situation, du type de trouble et des liens sociaux dont les intéressés disposent. L'enjeu est de garantir une continuité 
des soins ${ }^{22}$ et de rediriger dès que possible les patients vers le droit commun, à savoir le service public hospitalier classique, la psychiatrie de secteur ou la psychiatrie libérale, avec souvent des passerelles de l'une à l'autre. Les patients migrants hospitalisés sont orientés en fonction de I'initiale de leur nom de famille dans l'un des trois services psychiatriques du Bas-Rhin (EPSAN de Brumath, service de psychiatrie de l'hôpital civil de Strasbourg ou service de psychiatrie du centre hospitalier d'Erstein). Il est à noter, enfin, que le recours à I'interprétariat professionnel est très important au CMP Berne et à I'EMPP qui, depuis plusieurs années déjà, font appel aux interprètes de MSA $^{23}$. Identifiées comme des structures disposant de ressources en termes d'interprétariat, elles peuvent faire l'objet de logiques d'adressage impromptues de la part de médecins libéraux ou d'acteurs associatifs, leur adressant des patients ne relevant pas toujours d'une prise en charge psychiatrique ou du secteur concerné. Ces derniers sont alors à nouveau réorientés en conséquence par les équipes du CMP ou de l'EMPP.

À Strasbourg, à côté de ces prises en charge relevant de I'hôpital public, I'association Parole Sans Frontière (PSF) joue un rôle de première ligne dans le suivi thérapeutique des migrants dits allophones. Créée au tout début des années 1990, PSF résulte d'une volonté, portée par des psychologues et psychiatres, de proposer un soin psychothérapeutique aux populations migrantes non francophones. Mettant en place des séminaires de formation pour les internes en psychiatrie, PSF développe également des consultations gratuites, en présence d'interprètes, pour des patients venant de l'ensemble du Bas-Rhin. L'équipe est aujourd'hui constituée de quatre psychologues ${ }^{24}$ exerçant à temps partiel (salariés de l'association ou vacataires) et d'une psychiatre bénévole officiant par ailleurs en libéral. Bénéficiant du soutien de l'Agence régionale de santé, de la municipalité strasbourgeoise et des Nations unies notamment, PSF assure un suivi psychothérapeutique complémentaire, sur plusieurs points, au CMP et à I'EMPP. Tout d'abord, PSF a développé un volet spécifique de prise en charge pour les migrants victimes de torture et présentant des traumatismes liés aux conditions du parcours migratoire et/ou aux violences conjugales. L'activité de PSF répond aussi au souci d'éviter l'exclusion du soin de migrants présentant des troubles liés à la précarité, mais dont la prise en charge ne relèverait pas de dispositifs publics. La structure accueille également des patients dans un premier temps pris en charge par I'EMPP, mais qui, suite à un relogement, sont redirigés vers un CMP de secteur, parfois éloigné du centre-ville ou même de Strasbourg. Préférant une prise en charge en centre-ville, certains patients vont alors s'adresser à PSF dont l'activité n'est pas contrainte par la sectorisation.

22 Le transfert vers les CMP de secteurs peut néanmoins impliquer des ruptures dans la continuité des suivis thérapeutiques, ce que regrettent certains professionnels de la santé mentale y voyant un effet contre-productif de la sectorisation.

23 Dans le hall d'entrée de l'établissement, faisant office de salle d'attente en face du secrétariat, une affiche rédigée en français rappelle que les interprètes officiant sont mis à disposition par MSA, et que le coût horaire de l'interprétariat est pris en charge par I'hôpital. II est demandé de prévenir au moins vingt-quatre heures à l'avance en cas d'annulation de la consultation. L'affiche rappelle également la possibilité de demander le même interprète pour les différents rendez-vous, ou de changer si le besoin en est ressenti (par exemple, préférence pour un interprète homme ou femme, etc.).

24 Parmi eux, certains peuvent assurer des consultations en anglais, en turc, en allemand, voire en russe. 
Les logiques d'adressage vers PSF relèvent de plusieurs canaux. Si le bouche-à-oreille entre compatriotes joue un rôle important, certains migrants sont orientés par des réseaux partenaires, comme des structures d'aide aux demandeurs d'asile ou aux sans-papiers. Ces orientations peuvent être motivées par des enjeux territoriaux, marqués par la volonté de contourner la sectorisation et, possiblement, une rupture dans la continuité des prises en charge suite à un relogement. Elles peuvent aussi être motivées par la spécificité du suivi psychothérapeutique proposé par PSF pour les migrants victimes de torture et de violences. Le travail en réseau ${ }^{25}$ est ici central, à la fois dans le système d'aiguillage, mais aussi dans les modalités de prise en charge dans une perspective appréhendant la santé de manière globale. D'ailleurs, entre 2014 et 2015, PSF a consacré une fenêtre horaire pour des consultations ciblées sur des mineurs non accompagnés, dont nombre d'entre eux étaient hébergés en hôtel faute de place en foyer. En l'absence d'un suivi socio-éducatif adéquat, PSF a décidé d'interrompre ces prises en charge arguant du fait que le suivi psychothérapeutique ne pouvait se faire de manière satisfaisante dans ces conditions. En définissant une échelle d'action élargie par rapport à la sectorisation psychiatrique, PSF participe de la construction de nouveaux espaces sanitaires même si son activité peut rencontrer des limites liées à la nécessité d'une prise en charge globale. Qui plus est, PSF n'est pas en mesure d'assurer le suivi des migrants nécessitant une prise en charge institutionnelle en cas de maladie mentale.

La focale sur les dispositifs d'interprétariat dans le champ de la santé mentale à Rennes et Strasbourg participe à éclairer I'hétérogénéité des prises en charge des migrants en France. Cette hétérogénéité (Hoyez, 2011; D'Halluin et Hoyez, 2012), liée à la difficulté d'uniformiser des parcours de soins traversés par des enjeux non médicaux (papiers, logement, isolement social, etc.), est redoublée par le développement inégal de l'interprétariat selon les territoires. En outre, l'existence d'initiatives proactives en matière d'interprétariat sur un territoire donné ne signifie pas que tous les professionnels soient enclins à travailler avec un interprète auprès de ces populations.

\section{Des réticences dans le paysage professionnel}

Les réticences de certains professionnels de la santé mentale à prendre en charge la population migrante peuvent tenir autant au profil de cette patientèle qu'à la situation d'interprétariat.

Tout d'abord, le côté chronophage de la mise en place d'une consultation avec un interprète (formulaire spécifique à remplir auprès de la structure pourvoyeuse; impératif de disponibilité commune entre interprète et patient, mais aussi étirement de la durée de la consultation, etc.) peut constituer un frein à l'acceptation de cette patientèle. Les contraintes organisationnelles sont accentuées par l'effort de rationalisation du coût de l'interprétariat impliquant, pour les professionnels, d'essayer de programmer, dans une même demi-journée, plusieurs rendez-vous pour des patients différents avec un même interprète.

25 PSF est aussi très en lien avec le réseau RESPIRE, regroupant des thérapeutes sensibilisés à la question de la prise en charge de patients avec interprètes. 
À ces facteurs s'ajoutent des considérations d'ordre politique. D'une part, est parfois pointé le risque d'un déséquilibrage dans la patientèle si la population migrante en vient à prendre une place trop importante, mettant alors à mal l'universalité du système de santé et la mission de service public pour tous. Certains professionnels, de surcroît, craignent une disqualification de l'exercice de leur métier par un effet de postérité inopportune susceptible de glisser sur eux à partir des stigmates qui pèsent sur leur patientèle étrangère. $\mathrm{D}^{\prime}$ autre part, le suivi psychothérapeutique des migrants en situation de précarité est parfois décrit comme inefficace et entravé par l'incertitude administrative, lorsqu'ils sont sans-papiers ou dans l'attente d'une décision concernant leur demande d'asile. Un infirmier en psychiatrie exerçant en EMPP fait ainsi état des "difficultés" pointées par des confrères à ce sujet :

"Quand on travaille sur le syndrome post-traumatique, il y a certaines personnes qui veulent que la personne soit en situation régulière pour qu'il n'y ait pas de parasite dans la situation au quotidien, notamment avec tout ce qui concerne l'hébergement, l'argent.

C'est une réalité : les personnes qui sont préoccupées par le quotidien ne sont pas forcément en capacité de réfléchir sur le syndrome post-traumatique.» (Entretien, 2016)

À ses yeux, la condition de régularité du séjour et/ou d'hébergement stable requise par certains confrères entre dans la catégorie de refus de soins insidieux. Mais c'est aussi la question de l'instrumentalisation des soins voire de leurs détournements pour des raisons de "papiers" qui peut être mise en débat. Les représentations tenant à l'image du "migrant économique profiteur", qui se retrouvent dans d'autres contextes de soins (Pian, 2012 et 2016), peuvent alimenter des réticences à prendre en charge ce public. Ces représentations soulignent l'imbrication complexe entre politiques d'immigration et santé publique, déjà relevées depuis les années 1990 (Fassin, 1999; Fassin et D'Halluin, 2005; Musso, 2012).

Par ailleurs, selon certains psychologues et psychiatres, cette population nécessiterait une prise en charge spécifique liée aux évènements traumatiques vécus dans leur pays d'origine ou sur les routes migratoires, ce qu'ils ne seraient pas en mesure d'assurer. D'aucuns se sentiraient insuffisamment préparés à être confrontés à des contenus estimés "trop angoissants" et qui nécessiteraient, pour le thérapeute, un travail "à côté». L'argument politique croise à nouveau un argument thérapeutique auquel s'adossent I'appréhension ou le refus de travailler avec des interprètes. Ces réticences font intervenir des considérations "dogmatiques" (pour reprendre les termes d'un psychologue) liées à l'introduction d'un tiers dans la relation thérapeutique : "Ce n'est pas la culture psy de travailler à trois comme ça», souligne, en ce sens, un infirmier exerçant en EMPP. Ces considérations se mêlent à des enjeux pratiques attenants à ne pas "savoir comment faire pour travailler avec un interprète" : présence qui, de fait, vient modifier la dynamique des échanges.

En revanche, les praticiens très investis dans la prise en charge de la patientèle migrante présentent des trajectoires professionnelles et biographiques marquées par des points communs. Ils ont connu des expériences de vie à l'étranger ou ont connu personnellement ou via leur histoire familiale l'expérience de la migration en France. Ils ont été formés, quand bien même ils ont pu ensuite y porter un regard critique, à l'ethnopsychiatrie ou à la clinique 
transculturelle. Et ce, que ce soit lors de leur cursus initial ou d'expériences professionnelles antérieures à l'étranger (ont été mentionnées par exemple les Antilles, l'Afrique, la Roumanie), présentées comme fondatrices de leur intérêt pour l'altérité dans leur exercice professionnel. Enfin, ils se distinguent par une sensibilisation à la cause des étrangers ${ }^{26}$, parfois marquée par un engagement militant, comme en témoigne l'ouvrage paru en 2017, Migrants, le cri de Strasbourg au sujet de I'hospitalité des réfugiés en Europe.

Les paysages sanitaires décrits ici restent marqués par des réticences et des épreuves que relèvent les professionnels. II en résulte une influence sur les orientations et les logiques d'aiguillage, car les structures et les professionnels acceptant de travailler avec des interprètes sont vite identifiés et assignés à ce rôle. Quels regards les interprètes portent-ils, dans ce contexte, sur leurs interventions?

\section{Les épreuves de la consultation}

Selon les interprètes rencontrés, les difficultés de l'interprétariat en santé mentale ne se limitent pas à la dimension émotionnelle, à laquelle certains psychologues et psychiatres sont d'ailleurs attentifs, réservant à la fin de leurs consultations un rapide temps de "débriefing". Les difficultés résident plus largement, à leurs yeux, dans les sollicitations auxquelles ils sont confrontés.

\section{Les débats autour du « mot à mot »}

Lorsque les interprètes comparent leurs interventions dans le secteur de la santé mentale avec les autres domaines dans lesquels ils exercent, ils insistent de manière récurrente sur les exigences particulières en matière d'interprétariat qui leur sont apposées. Un interprète souligne combien "les psys attachent beaucoup d'importance à chaque mot» :

"Et ça m'est arrivé qu'un psychologue me demande : est-ce qu'il a dit ce mot-là?

Traduire un mot à part, ça n'existe pas, il faut traduire dans le contexte et parfois dans

le contexte je suis obligé de changer les mots, avec d'autres mots qui n'ont pas été prononcés. Et ça, c'est difficile dans le domaine psy. Il faut être fidèle au sens ou au mot? Si on arrive à faire les deux, c'est bien, sinon [...]. Attacher le sens au mot, oui, mais il ne faut pas trop. C'est pourquoi en psy, c'est très difficile l'interprétariat." (Interprète femme, russophone, 2015)

Dans le même temps, plusieurs interprètes font part de la difficulté à traduire des propos souvent décousus, ce qui nécessite une "gymnastique des langues" (Sinha, 2011) particulièrement épuisante ${ }^{27}$ : sur le ton de l'humour, un interprète exerçant depuis une dizaine d'années estime qu'il faut "être soi-même psychologue et apprenti sorcier" pour parvenir à tenir le rôle d'interprète dans de telles conditions. Or, traduire des propos décousus comporte une double contrainte. Lorsque les interprètes $s^{\prime}$ attachent à restituer le plus fidèlement possible le discours du patient, il peut y avoir malentendu sur la qualité de leur prestation

26 Ou plus largement par des formes d'engagement à destination des populations dites vulnérables.

27 À cela s'ajoutent les difficultés liées à la sphère de ce qui est appelé les «intraduisibles». 
et donc sur leurs compétences, dès lors que le professionnel a du mal à saisir le message transmis :

"II y a des professionnels qui te fixent, comme un hibou parfois, pendant que tu traduis. Il fixe son regard, il mesure le temps et le nombre de paroles prononcées [...].

Plutôt les psychiatres et pire encore, les psychologues. Je ne sais pas pourquoi ces deux catégories de professionnels font très attention. Peut-être qu'ils n'ont pas confiance en nos compétences pour tout traduire en bon français, dans la même structuration que la leur. Ou parce que le langage du psychologue et du psychiatre est tellement complexe."

(Interprète homme, arabophone, 2016)

Plusieurs psychologues et psychiatres ayant une habitude de travail avec les interprètes nuancent toutefois cette exigence en ce qui concerne le mot à mot.

" Je me suis déjà retrouvé avec des interprètes face à quelqu'un qui était délirant.

L'interprète était très embêté parce que le discours était incohérent donc ce qu'il me retransmettait [l'était aussi]. À un moment, il me dit: "Je traduis juste ce que j'entends". J'ai dit "Oui c'est ce que je te demande", il faut le leur dire parce que même si ce qu'ils entendent ne leur paraît pas cohérent, il faut nous le retraduire parce c'est ce qui va nous permettre de voir [les pathologies]. " (Infirmier exerçant en EMPP, décembre 2016)

La plupart disent adopter une posture assez souple à l'égard des interprètes, leur laissant s'emparer de leur rôle à leur façon. Les professionnels rencontrés insistent néanmoins sur l'importance d'un interprétariat sans reconstruction ni transmission de gestuels ou d'émotions venant "parasiter» leur travail. Pour une psychologue, l'interprétariat implique d'accepter de ne travailler qu'avec le signifiant (le mot traduit) et moins sur le signifié (les "tournures de phrases qui viennent signer une psychopathologie»). Cette même professionnelle insiste sur la nécessité de pouvoir distinguer ce qui vient du "psychisme des interprètes qui est en cours" parce que, pointe-t-elle, "forcément, ils interprètent quelque chose". Et ce, d'autant plus lorsque I'histoire du patient fait écho à leur subjectivité. Aussi, si pour certains psychiatres, l'interprète "arrivant à s'oublier" et à "utiliser le je" répond idéalement à ce qui est attendu, cette manière de traduire n'est pas nécessairement imposée. À cet égard, dans ses travaux, Wang (2016) montre comment cette oscillation entre le "je" et le "il/elle" est source de malaise pour les interprètes, qui cherchent à restituer la parole des patients sans pour autant se convertir en médiateur. Ce flou dans les échanges peut les conduire à "confondre les destinataires des questions du psychiatre" (ibid. : 143) ou du moins les oblige à composer avec de nombreux doutes.

Pour les professionnels rencontrés, la construction d'une relation de confiance tripartite est dite essentielle pour la dynamique thérapeutique. Certains insistent sur l'importance de recourir au même interprète durant toute la durée d'une prise en charge, dès lors que ce dernier participe du "continuum porté ensemble de consultation en consultation ". Une trop grande proximité est cependant décrite comme pouvant générer des effets pervers : un risque est que le patient devienne "passif» dans les échanges, s'en remettant à l'interprète. La "fidélisation" peut aussi conduire les interprètes à endosser un rôle d'avocat en formulant, au début ou à l'issue de la consultation, une requête pour le patient, ce qui n'est pas toujours bien perçu par les praticiens. 
$\mathrm{Si}$, dans les discours des professionnels de santé mentale rencontrés, les exigences à l'égard des interprètes apparaissent donc modulables, certaines pratiques ne sont pas tolérées. C'est le cas lorsque les interprètes posent directement des questions au patient pour lui demander des précisions ou explications, sans passer par le thérapeute. Ces pratiques font l'objet d'un rappel à l'ordre de la division sociale et morale du travail impliquant des rapports hiérarchiques et de pouvoir (Leanza et al., 2010) dans l'espace de la consultation. Cette division est également mise à l'épreuve par les apports en termes d'éclairages culturels ${ }^{28}$ des interprètes.

\section{"On n'est pas [seulement] des robots de la traduction"}

Des psychiatres perçoivent les interprètes comme de potentiels "décodeurs d'indices" leur permettant de mieux contextualiser les propos du patient : ils peuvent ainsi leur demander leur avis sur l'origine rurale ou urbaine du patient au regard du langage utilisé; ils peuvent également leur demander des précisions concernant l'organisation de la scolarité dans le pays d'origine ou encore l'interroger sur la spécificité de tel ou tel établissement scolaire. Selon certains professionnels rencontrés, manifester de l'intérêt pour la culture du patient peut en outre contribuer à libérer sa parole en lui donnant "une assise narcissique plus forte" (entretien, psychologue, 2016). De plus, ces apports culturels permettent d'éviter de considérer comme délirante la parole du patient alors qu'elle fait référence à des coutumes ou traditions du pays d'origine.

Or, c'est parfois l'interprète qui prend l'initiative de ces apports, d'autant plus lorsqu'une habitude de travail le lie au thérapeute, et qu'il sait que ce dernier est ouvert à ce type d'intervention. En témoigne la scène suivante, rapportée par une interprète russophone :

" J'avais un suivi psychologique, où la dame disait que par rapport à son premier mariage, elle avait été volée par son mari, qu'elle ne connaissait même pas, elle l'a vu une fois son mari [...] $]^{29}$. Donc, je traduis ça et le psychologue avait l'air perdu, parce que vous imaginez aujourd'hui on vous dit en France voilà : "Vous ne l'avez jamais connu la personne et puis vous sortez dehors, on vous vole, vous êtes obligée de rester toute votre vie avec cette personne, avoir des enfants et une famille". [...] Donc je me suis dit qu'il faut éclaircir cette situation. II m'a regardé parce que là, il ne comprenait plus. C'est la dame qui était délirante ou c'était vraiment... Donc, je me dis que c'est vrai que cette tradition existait en Géorgie et peut-être aujourd'hui c'est plus rare, mais il y a vingt ans c'était encore à la mode [...]. Je ne vais pas jouer à ce rôle que le psychologue pense que c'est la patiente qui est délirante et qu'elle invente des choses alors que je sais que ça existe vraiment dans le pays."

Ici, I'interprète a anticipé, au regard de sa "définition de la situation" (Thomas et Swain, 1928), une demande et une nécessité d'explications culturelles au sujet d'une pratique en vigueur dans le pays d'origine de la patiente, qui est aussi le sien. Elle justifie son initiative (soumise toutefois à l'approbation

28 Nous employons le terme de culture en nous démarquant d'une approche culturaliste, à I'instar des analyses en termes de relations inter-ethniques. Voir Cuche (1996) et Simon (2006).

29 II est fait référence à l'enlèvement ou rapt de la fiancée, pratique qui a lieu dans le Caucase notamment. 
du psychologue puisqu'elle lui demandera au préalable s'il est intéressé par une parenthèse culturelle) pour éviter des incompréhensions dans la relation de soin dont elle s'attribuerait la responsabilité.

Dans le cadre de leurs travaux sur l'interprétariat en santé mentale, Chambon et Carbonel (2015) relèvent que les interprètes sont confrontés à une "autonomie malgré (eux) $1^{30}$, du fait de la flexibilité de leur rôle qui, en situation, n'est pas toujours clairement défini. De plus, ils se retrouvent fréquemment confrontés à des exigences contradictoires entre d'une part, les attentes ou sollicitations des professionnels qui ont recours à leur service et d'autre part, les prescriptions de leur employeur, pour qui l'endossement d'un rôle de "médiateur culturel " peut être vu comme outrepassant les frontières du métier. Ainsi, du point de vue de $\mathrm{MSA}^{31}$, loin d'exercer une fonction de médiation, l'interprète doit construire une posture distancée du contexte émotionnel du patient. Sans pour autant jouer le rôle d'expert de la culture des patients étrangers, il est invité à exercer ses fonctions dans une attention interculturelle lui permettant de repérer d'éventuels quiproquos. Or, dans le cadre des interactions, la frontière entre la médiation culturelle et l'attention interculturelle peut vite être glissante.

Enfin, il est à noter que les professionnels de santé mentale rencontrés adoptent un positionnement différencié à l'égard de ces apports culturels. Certains sont plutôt ouverts à ces précisions, même s'ils adoptent une vigilance particulière quant à la manière de les prendre en compte dans le suivi thérapeutique. D'autres sont par principe très réticents, soulignant le fait que ces savoirs sont nécessairement situés, et que les interprètes ont parfois émigré depuis longtemps, les rendant éloignés de ce que serait la culture de leur pays $\mathrm{d}^{\prime}$ origine. Ces réserves se retrouvent aussi du côté de certains interprètes même si, pour d'autres, ces apports sont perçus comme valorisants : "on n'est pas des robots de la traduction", souligne ainsi l'un d'eux. Ne pas être un robot, c'est à leurs yeux prêter attention aux malentendus, humaniser la relation, s'ajuster en conséquence, voire tenter de revendiquer une expertise.

\section{Conclusion}

En abordant l'interprétariat en santé mentale sous l'angle de la division sociale, morale et spatiale du travail, l'article a permis d'articuler deux entrées portant sur les acteurs et les territoires porteurs de dispositifs. Ce faisant, il offre une double grille de lecture : la première, sur les inégalités socio-territoriales de santé au prisme des enjeux migratoires; la seconde sur les négociations se jouant, dans ce contexte, au cœur de la dynamique thérapeutique. Cette approche pluridisciplinaire permet de resituer la manière dont les professionnels de la santé mentale et les interprètes conçoivent leurs pratiques et "juste place" respectives, tout en rendant compte de la structuration du paysage sanitaire local. Dans le même temps, l'attention portée à la dimension socio-spatiale permet de mieux comprendre comment les pratiques et organisations professionnelles s'organisent et se distinguent : les approches différenciées révélées

30 "Lui» dans la citation.

31 Voir notamment la plaquette sur l'interprétariat professionnel produite en 2015 par MSA. 
sur nos terrains trouvent leurs fondements dans des logiques sociales et territoriales touchant autant à la dynamique des liens professionnels qu'aux modalités d'accueil des migrants à l'échelle locale.

Plus largement, l'interprétariat en santé mentale nous semble alors fonctionner comme fait révélateur, susceptible d'exercer une fonction miroir dans le cadre de la prise en charge des migrants. À l'instar du personnel scolaire et des intervenants sociaux, le personnel soignant est pris, dans une certaine mesure, dans l'apparente neutralité de la nouvelle catégorie institutionnelle $d^{\prime}$ " allophones" laquelle n'est pas sans lien avec les politiques contemporaines d'immigration et de l'asile (voir Armagnague-Roucher et al., 2018; ArmagnagueRoucher et Tersigni, 2019). Interroger les dispositifs d'interprétariat en santé mentale croise en ce sens enjeux thérapeutiques et politiques.

Cela dit, trois dimensions mériteraient d'être prolongées davantage. Tout d'abord, il pourrait être intéressant de creuser, au sein du domaine de la santé mentale, les enjeux spécifiques de l'interprétariat au regard des suivis psychiatriques, infirmiers ou psychologiques, mais aussi des suivis effectués par les travailleurs sociaux exerçant au sein des EMPP et CMP. Ensuite, sans se limiter à mettre en perspective les mobilités internationales des interprètes avec leur trajectoire professionnelle, il s'agirait, en changeant l'échelle d'analyse pour aller vers la prise en compte des territoires du quotidien, d'associer leur professionnalisation avec les mobilités urbaines et extra-urbaines, qui sont propres à l'exercice de leur métier. Enfin, un prolongement possible consisterait à élargir la perspective pluridisciplinaire ici présente, en introduisant les échelles temporelles dans l'analyse. Cette démarche, à partir notamment d'un travail d'archives, gagnerait à analyser dans une perspective longitudinale l'impact de la présence des interprètes dans la manière de remplir les dossiers médicaux face à une patientèle migrante. 


\section{Références bibliographiques}

Arend Louise, Cadart Aurélien, Chabagno Lucie, Delepoulle Ndiaye Anna, Deseille Reynald, Gautier Anne, Guiffault Tiphaine, Moutou Karine, Thomin Émilie et Urcun Mélisande (2017) L'interprétariat médical et social, élément de la démocratie sanitaire, Rapport, Module Inter Professionnel de Santé Publique, Rennes, École des Hautes Etudes en Santé Publique, 53 p.

Armagnague-Roucher Maïtena, Cossée Claire, Mendonça Catherine, Rigoni Isabelle et Tersigni Simona (2018) Étude sur la scolarisation des élèves allophones nouvellement arrivés (EANA) et des enfants issus de familles itinérantes et de voyageurs (EFIV) (EVASCOL), Rapport de recherche, Défenseur des Droits, $418 \mathrm{p}$.

Armagnague-Roucher Maïtena et Tersigni Simona (2019 [à paraître]) L'émergence de l'allophonie comme construction d'une politique éducative : le traitement scolaire des enfants migrants en France, Émulations.

Beal Arnaud et Chambon Nicolas (2015) Le recours à l'interprète en santé mentale : enjeux et problèmes, Cahiers du Rhizome, 55, pp. 9-19.

Bessin Marc (2005) Le travail social est-il féminin?, in Jacques lon Éd., Le travail social en débat(s), Paris, La Découverte, pp. 152-169.

Bonny Yves, Bautès Nicolas et Gouëset Vincent (Dirs.) (2017) L'espace en partage. Approche interdisciplinaire de la dimension spatiale des rapports sociaux, Rennes, Presses Universitaires de Rennes, 360 p.

Bourdin Marie-Jo et Larchanché Stéphanie (2015) De l'interprétariat à la médiation en santé mentale : I'expérience du centre Françoise Minkowska, Les Cahiers du Rhizome, 55, pp. 48-54.

Chambon Nicolas et Carbonel Natacha (2015) L'interprète, un intervenant en santé mentale, Les Cahiers du Rhizome, 55, pp. 86-97.

Chambon Nicolas et Le Goff Gwen (2016) Enjeux et controverses de la prise en charge des migrants précaires en psychiatrie, Revue française des affaires sociales, 2, pp. 123-40.

Cognet Marguerite, Hamel Christelle, Sauvegrain Priscille et Gabarro Céline (2014) Migration et état de santé, in Jean-Yves Blum-Le Coat et Mireille Eberhard Éds., Les immigrés en France, Paris, La documentation française, pp. 133-146.

Coldefy Magali (2016) Les soins en psychiatrie : organisation et évolutions législatives récentes, Revue française des affaires sociales, 6, pp. 21-30.

Coldefy Magali et Le Neindre Charlène (2014) Les disparités territoriales d'offre et d'organisation des soins en psychiatrie en France : d'une vision segmentée à une approche systémique, Les rapports de I'IRDES, 558, [en ligne]. URL : http://www.irdes.fr/recherche/rapports/558-les-disparites-territoriales-d-offre-etd-organisation-des-soins-en-psychiatrie-en-france.pdf

Crenn Chantal et Kotobi Laurence (2012) Du point de vue de l'ethnicité, pratiques françaises, Paris, Armand Colin, 348 p.

Cuche Denys (1996) La notion de culture dans les sciences sociales, Paris, La Découverte, 128 p.

Demazière Didier (2009) Postface : professionnalisations problématiques et problématiques de la professionnalisation, Formation emploi, 108, pp. 83-90. 
De Rudder Véronique, Poiret Christian et Vourc'h François (2000) L'inégalité raciste. L'inégalité républicaine à l'épreuve, Paris, PUF, 224 p.

D'Halluin Estelle et Hoyez Anne-Cécile (2012) L'initiative associative et les reconfigurations locales des dispositifs d'accès aux soins pour les migrants primo-arrivants. Humanitaire, 33, [en ligne]. URL : http://humanitaire.revues. org/1407

Eliot Emmanuel, Lucas-Gabrielli Véronique et Mangeney Catherine (2017) Territorialisation sanitaire et décentralisation : état des lieux et enjeux à partir du cas français, Revue francophone sur la santé et les territoires, [en ligne] consulté le 10/07/2017. URL : https://rfst.hypotheses.org/eliot-emmanuel-lucas-gabrielliveronique-mangeney-catherine

Fassin Didier (Éd.) (2012) Les nouvelles frontières de la société française, Paris, La Découverte, $590 \mathrm{p}$.

Fassin Didier (2000a) Les politiques de l'ethnopsychiatrie, L'Homme, 153, [en ligne] consulté le 25/05/2018. URL : http://journals.openedition.org/lhomme/14

Fassin Didier (2000b) Repenser les enjeux de santé autour de l'immigration, Hommes et Migrations, 1225, pp. 5-12.

Fassin Didier (1999) Santé et immigration. Les vérités politiques du corps, Cahiers de I'Urmis, 5, [en ligne] consulté le 30/08/2018. URL : http://journals. openedition.org/urmis/351

Fassin Didier and D'Halluin Estelle (2005) The Truth in the Body. Medical certificates as ultimate evidence for asylum-seekers, American Anthropologist, 107 (4), pp. 597-608.

Hachimi Alaoui Myriam et Nacu Alexandra (2010) Soigner les étrangers en situation irrégulière. Des politiques migratoires aux postures professionnelles, Hommes et Migrations, 1284, pp. 163-173.

Hassenteufel Patrick (2008) Syndicalisme et médecine libérale : le poids de I'histoire, Les Tribunes de la santé, 18 (1), pp. 21-28, [en ligne]. URL : https://www. cairn.info/revue-les-tribunes-de-la-sante-2008-1-page-21.htm?contenu=article

Hoyez Anne-Cécile (2015) Territoires, mobilités et santé : comment composer et penser une offre de soins pour les populations migrantes en situation précaire?, Revue Francophone sur la Santé et les Territoires, [en ligne]. URL : https://rfst. hypotheses.org/hoyez-anne-cecile-territoires-mobilites-et-sante-comment-composer-et-penser-une-offre-de-soins-pour-les-populations-migrantes-en-situationprecaire

Hoyez Anne-Cécile (2011) L'accès aux soins des migrants en France et la "culture de l'initiative locale". Une analyse des contextes locaux à l'épreuve des orientations nationales, Cybergeo, [en ligne]. URL : http://cybergeo.revues.org/24796

Hughes Everett C. (1958) Men and their Work, Glencoe, The Free Press, 184 p.

Kessar Zahia (2000) La formation en anthropologie à l'hôpital, Hommes et Migrations, 1225, pp. 112-117.

Kotobi Laurence, Larchanché Stéphanie et Kessar Zahia (2013) Enjeux et logiques de recours à l'interprétariat en milieu hospitalier : une recherche-action autour de I'annonce d'une maladie grave, Migrations santé, 146-147, pp. 53-80. 
Laval Christian et Zeroug-Vial Halima (2015) L'interprétariat en santé mentale, Cahiers du Rhizome, 55, 101 p., [en ligne] consulté le 31/01/2017. URL : http:// www.ch-le-vinatier.fr/orspere-samdarra/rhizome/anciens-numeros/cahiers-derhizome-n55-I-interpretariat-en-sante-mentale-1058.html

Leanza Yvan, Boivin Isabelle and Rosenberg Ellen (2010) Interruptions and resistance: a comparison of medical consultations with family and trained interpreters, Social Science and Medicine, 70 (12), pp. 1888-1895.

Le Défenseur des droits (2016) Les droits fondamentaux des étrangers en France, Paris, $305 \mathrm{p}$.

L'information psychiatrique (2015a) Migrants (1/3), L'information psychiatrique, 91 (1), pp. 9-65, [en ligne]. URL : https://www.cairn.info/revue-I-informationpsychiatrique-2015-1.htm

L'information psychiatrique (2015b) Migrants (2/3), L'information psychiatrique, 91 (2), pp. 97-144., [en ligne]. URL : https://www.cairn.info/revue-l-informationpsychiatrique-2015-2.htm

L'information psychiatrique (2015c) Migrants (3/3), L'information psychiatrique, 91 (3), pp. 203-254, [en ligne]. URL : https://www.cairn.info/revue-I-informationpsychiatrique-2015-3.htm

Musso Sandrine (2012) Être régularisé au titre de la maladie en France, Corps, 10 (1), pp. 153-161.

OFII (2017) Rapport d'activité 2016, Office français de l'immigration et de l'intégration, $101 \mathrm{p}$.

ORIV (2017) Note Statistique de l'Observatoire Régional de I'Intégration et de la Ville (Immigrés-es et primo-arrivants-es dans le Grand Est), 6 p.

Payet Jean-Paul (1997) Le "sale boulot". Division morale du travail dans un collège en banlieue, Les Annales de la recherche urbaine, 75, pp. 19-31.

Pestre Élise (2010) La vie psychique des réfugiés, Paris, Payot et Rivages, 377 p.

Pian Anaïk (2016) Tourisme médical versus immigration thérapeutique : des catégories exogènes réductrices, Revue francophone sur la santé et les territoires, [en ligne]. URL : https://f.hypotheses.org/wp-content/blogs.dir/1946/files/2016/10/ Pian_rfst_2016_TMS.pdf

Pian Anaïk (2014) La fabrique de l'interprétariat auprès des immigrés atteints de cancer : la place des proches en, question in Florence Douguet, Françoise Leborgne-Úguen et Simone Pennec Éds., Les négociations du soin. Les professionnels, les malades et leurs proches, Rennes, PUR, pp. 193-204.

Pian Anaïk (2012) De I'accès aux soins aux trajectoires du mourir. Les étrangers atteints de cancer face aux contraintes administratives, Revue Européenne des Migrations Internationales, 28 (2), pp. 101-127.

Piret Bertrand et Douville Olivier (Dirs.) (2017) Migrants, réfugiés, la politique interroge la clinique, Psychologie clinique, 43 (1), pp. 5-6.

Plateforme d'accueil des Demandeurs d'Asile (2016) Compte-rendu narratifAnnée 2015 de la plateforme d'accueil des demandeurs d'asile de Rennes, 21 p.

Rechtman Richard (1992) L'intraduisible culturel en psychiatrie, L'évolution psychiatrique, 57 (3), pp. 347-365. 
Rhenter Helène et Rhenter Pauline (2012) Les ambiguïtés de la nouvelle territorialisation, in Lise Demailly et Michel Autès Éds., La politique de santé mentale en France. Acteurs, instruments, controverses, Paris, Armand Colin, pp. 141-152.

Rhenter Pauline et Michel Jacques (2010) Culture et identité : Ia psychiatrie publique à l'épreuve, in Michel Joubert et Claude Louzoun Dirs., Répondre à la souffrance sociale, Paris, Érès, pp. 117-135.

Simon Pierre-Jean (2006) Pour une sociologie des relations interethniques et des minorités, Rennes, Presses Universitaires de Rennes, 347 p.

Sinha Shumona (2011) Assommons les pauvres, Paris, L'Olivier, 149 p.

Tabouri Aziz (2009) L'interprétariat dans le domaine de la santé, Hommes \& Migrations, 1282 (6), pp. 102-107.

Thomas William Isaac and Swain Thomas Dorothy (1928) The Child in America. Behavior Problems and Programs, New York, Alfred Knopf, 553 p.

Veïsse Arnaud, Wolmark Laure, Revault Pascal, Giacopelli Maud, Bamberger Muriel et Zlatanova Zornitza (2017) Violence, vulnérabilité sociale et troubles psychiques chez les migrants/exiles, Bulletin Épidémiologique Hebdomadaire, 20-21, pp. 406-414.

Wang Simeng (2016) "Aidez-nous à comprendre vos Chinois!». Conditions de possibilités de la légitimation du sociologue en milieu psychiatrique, Genèses, 4 (105), pp. 141-156.

Wittorski Richard (2008) La professionnalisation, Savoirs, 2 (17), pp. 9-36. 


\section{Anaïk Pian, Anne-Cécile Hoyez et Simona Tersigni \\ L'interprétariat en santé mentale : divisions sociale, morale et spatiale du travail dans les soins aux migrants}

L'interprétariat dans la prise en charge des migrants en santé mentale est inséré dans de nombreux enjeux politiques qui interpellent les pratiques des professionnels à l'égard d'une patientèle dont souffrances psychiques et conditions de l'exil sont souvent entremêlées. Les consultations mettent également à l'épreuve les interprètes qui doivent se situer dans une relation de soin complexe, alors même qu'ils peuvent se retrouver face à des exigences contradictoires entre les attentes des professionnels de santé mentale et les prescriptions des associations qui les emploient. À travers le prisme de l'interprétariat, l'article interroge les divisions sociale, morale et spatiale du travail dans la prise en charge des migrants en santé mentale. II s'appuie sur deux terrains d'enquête à Rennes et à Strasbourg, deux capitales régionales marquées par une évolution des flux migratoires, et comptant des initiatives importantes en matière de promotion de l'interprétariat professionnel.

\section{Interpretation in Mental Health Services: Social, Moral and Spatial Divisions of Work in Migrant Care}

Simultaneous interpreting in the context of mental health care for migrants faces several political issues, which result in questioning such professional practices towards patients whose mental suffering is often related to the experience of exile. Consultation sessions with migrants represent a challenge for interpreters as well. These professionals are required to position themselves in a caring relationship, while dealing with mental health professionals' expectations together with employers' directives, which may overlap and contradict each other. Through the prism of interpretation, the paper aims at examining the social, moral and spatial division of labour in migrants' care, namely in the context of mental health services. The presentation is based on two separate field-works, in the cities of Rennes and Strasbourg: both regional capitals are marked by recent significant changes in migration flows and have been considered for experimenting innovative practices in promoting simultaneous interpretation in the context of mental health care.

\section{La interpretación en la salud mental: divisiones sociales, morales y espaciales en el trabajo de atención al migrante}

La interpretación en el cuidado de los migrantes en salud mental se inserta en muchos asuntos políticos que desafían las prácticas de los profesionales que se preocupan por un paciente cuyo sufrimiento mental y condiciones de exilio a menudo se entrelazan. Las consultas también desafían a los intérpretes que necesitan estar en una relación de cuidado compleja, a pesar de que pueden enfrentar demandas conflictivas entre las expectativas de los profesionales de la salud mental y las prescripciones de las asociaciones que las emplean. A través del prisma de la interpretación, el artículo cuestiona las divisiones sociales, morales y espaciales del trabajo en el cuidado de los migrantes, especialmente en la salud mental. Se basa en dos sitios de encuestas en Rennes y Estrasburgo, dos capitales regionales marcadas por una evolución de los flujos migratorios, y contando importantes iniciativas en la promoción de la interpretación profesional en salud mental. 